\title{
The effect of observational learning on the development of central sensitization
}

Torta $\mathrm{DM}^{1}$, Meyers $\mathrm{E}^{1}$, Polleunis $\mathrm{K}^{1}$, De Wolf $\mathrm{S}^{1}$, Meulders $\mathrm{A}^{1,2}$, van den Broeke $\mathrm{EN}^{3}$

${ }^{1}$ Health Psychology, Faculty of Psychology and Educational Sciences, KU Leuven, Leuven, Belgium

${ }^{2}$ Experimental Health Psychology, Faculty of Psychology and Neuroscience, Maastricht, The Netherlands

${ }^{3}$ Institute of Neuroscience, division Cognition and Systems, Faculty of Medicine, UCLouvain, Brussels, Belgium

Corresponding author:

Prof. Dr. Diana Torta, Phd

Tiensestraat 102, 3000 Leuven Belgium

diana.torta@kuleuven.be 


\begin{abstract}
Watching other people in pain may affect one's own experience of pain. It is unknown whether it can also modulate secondary mechanical hypersensitivity. We have addressed this question in two experiments in healthy human volunteers. In experiment 1 we tested, on a large sample $(\mathrm{N}=83)$, five videos of a model demonstrating high or low pain during high frequency stimulation (HFS) of the skin, a procedure known to induce secondary mechanical hypersensitivity. The aim was to select the two videos rated with the highest and lowest expected pain and fear (high pain and low pain videos). Morevoer, we have explored the correlation between empathy and fear scores. In experiment $2(\mathrm{~N}=44)$, two groups of participants were randomly allocated to watching either the low or the high pain video, and subsequently underwent HFS. The high pain video group reported increased pain during HFS. The two groups differed in the magnitude of secondary mechanical hypersensitivity after HFS, but the unpleasantness scores for mechanical stimulation after HFS, as well as spread of hyperalgesia were not statistically different. Empathy scores correlated positively with fear reports in experiment 1 but not experiment 2. Unexpectedly, we found higher scores of fear of pain for the high pain video only in experiment 1. In summary, observational learning of a model demonstrating high pain seems to have a stastistically significant but small effect on pinprick hypersensitivity. Its operating mechanisms remain partially elusive.
\end{abstract}




\section{Introduction}

Building on Bandura's observational learning theory (1), previous research has found that the observation of others in pain is an important source for the development of pain beliefs and fear (2-13). Indeed, several reports $(3,6,7,9,10,14-17)$ have indicated that the observation of others in pain can affect experimentally induced pain perception, and may impart significant placebo- nocebo effects, via expectations, but also purportedly via modulation of pain-related fear $(2,4,11,18)$.

Experimental pain, such as the topical application or injection of capsaicin and electrical stimulation of the skin, induces mechanical pinprick hypersensitivity of the surrounding skin (secondary hyperalgesia), which is considered to be a manifestation of central sensitization, i.e. the increased responsiveness of nociceptive neurons in the central nervous system (19). It is believed that central sensitization contributes to chronic pain $(20,21)$. Whether observational learning can modulate secondary mechanical pinprick hypersensitivity (or central sensitization) is yet unknown.

To address this question we conducted two experiments. In experiment 1 we showed participants five videos of an actress undergoing High-Frequency electrical stimulation of the skin (HFS). HFS is a procedure that, besides being painful, induces consistently across volunteers a robust pinprick hypersensitivity of the skin adjacent to the site at which HFS is applied. The videos differed on the perceived painfulness expressed and reported by the actress during HFS. After watching the videos, participants provided ratings of fear of HFS and expected pain intensity of the procedure. These results were then used to select, for experiment 2, the two videos showing the largest differences on fear and expected painfulness, the "high pain" and "low pain" video. In experiment 2, volunteers were randomly assigned to watch the "low pain" video, in which the actress demonstrated and reported mild pain ("low pain" group), or the "high pain" video, where the actress demonstrated reported intense pain ("high pain" group ). After having seen the videos, the participants underwent HFS themselves, and were tested for i) the presence and magnitude of pinprick hypersensitivity and its unpleasantness at several time points after applying HFS, ii) the vertical length of the area of pinprick hypersensitivity. Crucially, the videos only portrayed the 
actress in different degrees of pain during HFS, but did not provide any information about the subsequent development of pinprick hypersensitivity, which was our primary outcome. We hypothesized that the high pain video would induce more fear of HFS than the low pain video, leading to higher pain ratings during HFS and as a consequence to more pinprick hypersensitivity.

Additionally, we were interested in investigating the potential contribution of empathy to fear scores $(18,22)$. We hypothesized that the more the participants could identify with the model, the more the fear of pain they would develop. In experiment 1, we hypothesized that higher scores on the empathy scale would be associated with higher developed fear after watching a person in pain. In experiment 2, since our main hypothesis regarded an increase of fear in the high pain video, we planned to use empathy scores as a potential moderator in case of a significant relationship between fear and hyperalgesia.

\section{Materials and Methods}

\subsection{Participants}

Experiment 1 was performed online via Qualtrics $\mathrm{XM}^{\mathrm{OS}}$. One-hundred participants were recruited via snowballing techniques, but only 83 participants fulfilled the inclusion criteria (17 participants were older than 40 years, see also later).

Experiment 2 was performed in the laboratory. Forty-five participants were included. This number was based on a sample size calculation performed with MorePower 6.0.4 (23) using Cohen's $\mathrm{f}$ effect size of 0.4 for the interaction, standard 0.05 alpha error probability, power of 0.80. The f effect size was derived from a previous report by van den Broeke and colleagues (24) on the role of negative expectations on pinprick hyperalgesia. One participant dropped out, resulting in a final sample of 44 volunteers ( $N=22$ per group). This experiment was preregistered on the Open Science Framework (https://osf.io/mxsa5) prior to data acquisition.

Both experiments had been approved by the Social and Societal Ethics Committee of KU Leuven (G-2019 12 1893) and were conducted according to the Helsinki Declaration. Before the beginning of each study a written informed consent was obtained from each participant. Inclusion criteria were: 1) being female, 2) being 18 years of age or older but younger than 40, and 3) being a native or fluent Dutch speaker. Exclusion criteria were: 1) having participated 
in a study using High or Low Frequency stimulation of the skin, 2) having used paracetamol or other anti-inflammatory and/or painkiller $<12$ hours before the experiment, 3) having heart and vascular problems, 4) having respiratory or neurological diseases, 5) suffering from pain of a duration of 3 months or longer (chronic pain), 6) having a pacemaker or another electronical implant, 7) having uncorrected hearing and/or vision problems, 8) having psychiatric disorders, 9) under regular medication use (except anticonception), 10) being pregnant or sleep deprived ( $<5$ hours) at the moment of testing. Additional exclusion criteria for experiment 2 were: 1) having scars or tattoos on their ventral forearms and 2) presenting symptoms of Covid-19. We chose to recruit only women in order to prevent potential gender effects as both the experimenters ( $\mathrm{SdW}$ and KP) and the model in the video were women.

\subsection{Design of the study}

\subsubsection{Experiment 1}

In this within-subject experiment, participants were asked to watch and evaluate 5 online videos (see https://osf.io/mxsa5). On the day of testing, participants received a link to participate and were asked to fill in their demographics including age and level of education. After that, they were presented with the 5 videos; the presentation order was counterbalanced across participants.

In all videos the same female model (a young Caucasian amateur actress in her early 20s) pretended to undergo HFS on one of the two ventral forearms (see 2.3 for a description of HFS). HFS, when delivered at an intensity corresponding to $20 \times$ the electrical detection threshold to a single pulse, is experienced as painful in the majority of people (25) and induces increased pinprick sensitivity of the skin surrounding the site at which HFS is applied (24-35). In reality the actress received only very mild stimuli. The five videos differed on the perceived intensity of HFS pain as expressed by different facial expressions and bodily reactions to the stimulation and the pain ratings reported after each train. Each train was rated on a numeric rating scale (NRS) ranging from 0 (did not feel anything) to 100 (the most intense pain possible) with 50 being the anchor for the transition from non-painful to painful sensations. In the low pain videos (videos 1-2) the actress expressed mild reactions to the five trains, which were rated as 60-60-55-55-55. In the high pain videos (videos 4 and 5), she displayed intense pain and provided $80-80-85-85-85$ as ratings. Video 3 was used to test the effects of a neutral expression associated with low ratings (as in videos 1 and 2). All videos lasted approximately 
1 minute and were filmed in the same room where experiment 2 took place. After watching each video, participants answered the questions "How afraid would you be should you have to undergo this procedure" and "How painful do you expect the stimulation to be?" on a scale from 0 to 4, where 0 was not fearful/not painful, 1 was slightly fearful/painful, 2 was medium fearful/painful, 3 was very fearful/painful, 4 was extremely fearful/painful. In order to investigate whether these scores were more influenced by the facial and bodily expressions or by the ratings of the actress we further asked: "To what extent did the following aspects have an effect on your answers: The facial/bodily expressions that the woman showed, the pain ratings that the woman provided". Answers were given by moving a scroll bar from 0 to 10 for each of the two elements (facial/bodily expressions and ratings). Finally, participants were asked to fill in the Interpersonal Reactivity Index (IRI) questionnaire $(36,37)$, and empathy scale.

\subsubsection{Experiment 2}

In this between-subject experiment, participants watched either the "high pain" or "low pain" video (selected from Experiment 1) before they received HFS on one of the two ventral forearms. The day before their participation, participants were requested to fill in the following questionnaires online via Qualtrics; the Interpersonal Reactivity Index (IRI), the State-Trait Anxiety Inventory (STAI) (38,39), the modified Differential Emotions Scale (mDES) (40), the Pain Catastrophizing Scale (PCS) $(41,42)$, and the Fear of Pain Questionnaire-III (FPQIII) $(43,44)$. A description of the questionnaires can be found in the Supplementary material. The questionnaires were presented to control for potential psychological differences in the low and high pain groups. On the day of testing, at the beginning of the experiment, participants received a written standardized instruction (see Supplementary material) describing the procedure. After that, the baseline pain sensitivity (perceived intensity and unpleasantness) to mechanical pinprick stimuli was assessed on both forearms (measurement T0, see also 2.5). The arm that was stimulated first was counterbalanced across participants. The assessment of pinprick sensitivity was followed by the establishment of the detection threshold to a single electrical pulse (see also 2.4). After these baseline measurements, participants were randomly assigned to either a "high pain" or "low pain" group. In both groups, participants were told that before receiving HFS they first would watch a video showing the HFS procedure. The participants in the "high pain" group watched the high pain 
video selected in Experiment 1 (video 4) and the "low pain" group watched the low pain video (video 2). After watching the video, HFS was applied to one of the two ventral forearms (see 2.3) and participants were instructed to rate their perceived pain intensity for each train on the same NRS as the one used by the model in Experiment 1. The arm at which HFS was delivered (dominant or non-dominant) was counterbalanced across participants. After applying HFS, mechanical pinprick sensitivity was assessed immediately after HFS (T1) and 10 (T2), 20 (T3) and 45 (T4) minutes after HFS at the skin surrounding the site at which HFS was applied and at the same skin area at the contralateral control arm (see Figure 1). Following the last measurement of pinprick sensitivity the "spread" of pinprick hypersensitivity was established by measuring the distance (in $\mathrm{cm}$ ) between the proximal and distal borders of hypersensitivity from the centre of the concentric electrode. Finally, participants filled in an exit questionnaire, assessing fear ratings for HFS, the credibility of the model, for the influence of the model's facial expression and posture in the video, and the influence of the pain scores given in the video.

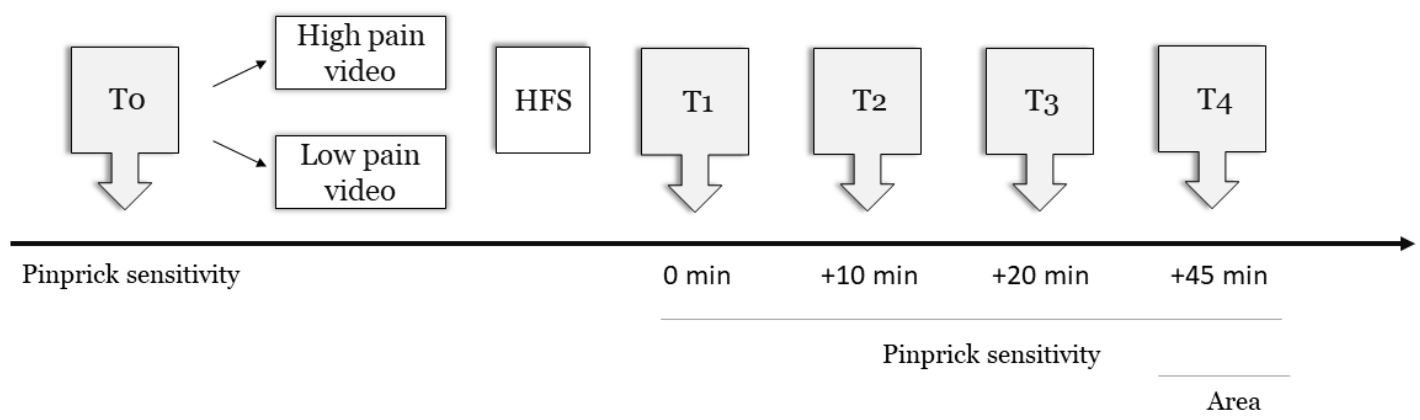

Figure 1. Timeline of experiment 2.

\subsection{High-Frequency electrical Stimulation (HFS)}

HFS consisted of five trains of $100 \mathrm{~Hz}$ electrical stimuli (pulse width $2 \mathrm{~ms}$ ) lasting $1 \mathrm{~s}$ each and repeated in a 9 second inter-train interval. HFS was delivered at an intensity corresponding to $20 x$ the individual detection threshold (see 2.4.) to a single electrical pulse (pulse-width $2 \mathrm{~ms}$ ). The HFS protocol was programmed in Matlab, generated with a DS5 (Digitimer) electrical stimulator and delivered to the skin via a custom-build concentric electrode. The electrode consisted of 16 blunt stainless steel pins with a diameter of $0.2 \mathrm{~mm}$ protruding $1 \mathrm{~mm}$ from the base. The pins formed a circle of $10 \mathrm{~mm}$ and constitute the cathode. The anode, made also of 
stainless steel, surrounded concentrically the anode, and had an inner diameter of $22 \mathrm{~mm}$ and an outer diameter of $40 \mathrm{~mm}(25,45,46)$.

\subsection{Electrical detection thresholds}

Single electrocutaneous stimuli ( $2 \mathrm{~ms}$ ) were administered one by one, starting at $0.1 \mathrm{~mA}$ with increasing steps of $0.1 \mathrm{~mA}$. Once the stimulus was detected, stimuli were presented in decreasing steps of $0.05 \mathrm{~mA}$ until the stimulus was no longer perceived, after which the intensity increased again in steps of $0.025 \mathrm{~mA}$. After three reversals, the detection threshold was established.

\subsection{Mechanical pinprick sensitivity}

To assess mechanical pinprick sensitivity a calibrated hand-held $128 \mathrm{mN}$ pinprick stimulator (MRC Systems GmbH, Mannheim, Germany) was used.

\subsubsection{Perceived mechanical pinprick intensity and unpleasantness}

Participants were asked to rate three mechanical pinprick stimuli applied at $1.5 \mathrm{~cm}$ from the center of the concentric electrode on an NRS ranging from 0 ("no sensation at all") to 100 ("extremely painful") with 50 being the anchor for the transition from non-painful to painful sensations. The perceived unpleasantness of the sensation was rated on an NRS ranging from 0 ("not unpleasant at all") to 100 ("as unpleasantness as possible"). Afterwards, the average of the three ratings was calculated to use for analysis.

\subsubsection{Determining the spread (vertical length) of increased pinprick sensitivity}

To map the spread of increased pinprick sensitivity after HFS the pinprick stimulator was applied onto the skin every $1 \mathrm{~cm}$ from the wrist and cubital fossa towards to center of the concentric electrode until the point at which the participant noticed a clear increase in pinprick sensitivity. The proximal and distal borders of increased pinprick sensitivity induced after HFS have been shown to be very reliable (47).

\subsection{Statistical analysis overview}

The statistical analyses were performed in SPSS (version 28, IBM Statistics).

\subsubsection{Experiment 1}


To test for statistical significant differences in the ratings of fear and expected pain intensity across the 5 videos we conducted a non-parametric Friedman test due to the ordinal nature of the scale. Significant tests were followed up with a Wilcoxon signed-rank test. Our aim was to select the two videos showing the largest difference in fear and expected painfulness. We used a 2-tailed Spearman's Rho correlation to unveil a potential relationship between empathy scores and fear ratings. Empahty scores, calculated as total scores of the IRI, were correlated to fear ratings for each video, and a correction for multiple comparisons was applied.

\subsubsection{Experiment 2}

\subsubsection{Primary outcomes}

The statistical analyses for Experiment 2 were conducted according to our pre-registered statistical plan (see https://osf.io/mxsa5). For the first two primary outcome measures, the perceived intensity and perceived unpleasantness of the mechanical pinprick stimuli, we conducted two separate repeated measures (RM) ANOVAs with "TIME" (T0, T1, T2, T3 and T4) and "SIDE" (HFS arm vs. control arm) as within-subject factors and "GROUP" (low pain vs. high pain) as between-subject factor. We hypothesized a statistically significant TIME $\mathrm{x}$ SIDE $\mathrm{x}$ GROUP interaction revealing a larger increase in mechanical pinprick sensitivity after HFS at the HFS-treated arm for the "high pain" group compared to the "low pain" group. Regarding the second primary outcome measure, the proximal-distal length of the "spread" of increased pinprick sensitivity after HFS, we hypothesized that the "high pain" group would show on average a larger proximal-distal length compared to the "low pain" group. To test this we performed a one-tailed independent samples t-test on the proximal-distal length.

\subsubsection{Secondary outcomes}

As secondary outcomes we tested whether the "high pain" group rated the HFS trains as more painful compared to the "low pain" group. For this, we conducted a one-tailed independent samples t-test on the average of the pain ratings obtained after each train in both groups. We further tested if there was a relationship between the fear of HFS as measured by fear ratings and the increased pinprick sensitivity induced by HFS, and whether this potential relationship was moderated by empathy scores. The increased pinprick sensitivity was calculated as the difference between the difference score (post minus pre HFS) of the two arms per time point. 
The two groups were also compared in terms of scores on the exit questionnaire by using the non-parametric Mann-U Whitney test and psychological questionnaires by using independent samples t-tests.

\section{Results}

\subsection{Experiment 1}

\subsubsection{Empathy}

The average total score $( \pm S D$ ) of the Interpersonal Reactivity Index (IRI) questionnaire was $88.06( \pm 7.81)$ (range 28-140).

\subsubsection{Fear of pain}

Overall, participants reported a median score of 2.2 (moderately) for the "low pain" video and of 3.2 for the "high pain" videos. The difference was also statistically significant $\left(X^{2}=252.84\right.$, $\mathrm{p}<0.0001)$. More specifically, differences were observed between low and high pain videos 1 vs. 4: $Z=-7.641, p<0.0001$; video 1 vs. video $5 Z=-7.786, p<0.0001$; video 2 vs. video 4: $Z=-8.674$, $p<0.0001$; and video 2 vs. video $5 Z=-8.587 p<0.0001$. There was no statistically significant difference between videos 1 to 3 and the videos 4 and 5 .

\subsubsection{Expected painfulness}

Participants reported a median score of 2.5 for the low pain video and 3.7 for the high pain video. The difference was significant $\left(X^{2}=327.5, p<0.0001\right)$, with again videos 1 and 2 differing from 4 and 5 (video 1 vs. video 4: $Z=-8.468, p<0.0001$; video 1 vs. video: $5 Z=-8.276, p<0.001$; video 2 vs. video 4 : $Z=-8.732, p<0.001$, video 2 vs. video $5 Z=-8.636 p<0.0001$ ).

Please note that these differences remained statistically significant even after randomly choosing 15 or $20 \%$ of the original sample. This was due to ensure that statistical differences held also for smaller sample sizes as those that we planned to use for experiment 2.

\subsubsection{Correlation between empathy and fear}

We observed statistically significant correlations between empathy and fear ratings for both the low pain videos ( 1 and 2$)$, and high pain videos ( 4 and 5$)\left(r_{s(v i d e o 1)}=0.216 p=0.028\right.$, $r_{s(\text { video })}=0.190 p=0.005, \quad r_{s(\text { video3 })}=0.118 p=0.234, \quad r_{s \text { (video4) }}=0.219 \quad p=0.027, \quad r_{s(\text { video })}=0.294$ 
$\mathrm{p}=0.003)$. Only the correlation for video 5 remained significant after correcting for multiple comparisons.

\subsubsection{Correlation between pain expectations and fear}

Strong statistically significant correlations were observed between scores of fear and expected pain in all conditions (video 1: $r_{s}=0.608, p<0.001$; video 2: $r_{s}=0.638, p<0.001$; video 3: $r_{s}=0.662$, $p<0.001$; video 4: $r_{s}=0.662, p<0.001$; video $\left.5: r_{s}=0.720, p<0.001\right)$. All correlations survived the correction for multiple comparisons.

\subsection{Experiment 2}

\subsubsection{Psychological questionnaires}

No statistically significant differences were observed regarding anxiety, fear of pain and pain catastrophizing between the two groups (see Table 1).

\begin{tabular}{|c|c|c|c|c|c|}
\hline & $\begin{array}{l}\text { Low pain } \\
\text { group }\end{array}$ & $\begin{array}{l}\text { High pain } \\
\text { group }\end{array}$ & t-test value & $p$ value & $\begin{array}{l}\text { Effect size } \\
\text { (Cohen's d) }\end{array}$ \\
\hline$I R I$ & 77.45 (8.17) & $75.63(9.93)$ & 0.646 & 0.522 & 0.195 \\
\hline STAI & $45.22(9.46)$ & 42.13 (10.59) & 1.021 & 0.313 & 0.308 \\
\hline mDES positive & $39.50(4.76)$ & $39.40(4.82)$ & 0.063 & 0.950 & 0.019 \\
\hline $\begin{array}{l}\text { mDES } \\
\text { negative }\end{array}$ & $23.45(6.83)$ & $22.50(6.06)$ & 0.490 & 0.627 & 0.148 \\
\hline PCS & $18.81(7.63)$ & $17.27(9.39)$ & 0.599 & 0.553 & 0.180 \\
\hline$F P Q-I I I$ & $14.95(5.06)$ & $16.13(4.73)$ & -0.799 & 0.429 & -0.241 \\
\hline
\end{tabular}

Table 1. Mean ( $\pm S D)$ scores and statistical comparison for psychological questionnaires. IRI: Interpersonal Reactivity Index (global score, empathy), STAI (anxiety), mDES (modified differential emotions scale), PCS Pain catastrophizing scale, FPQ-III Fear of pain questionnaire. Effect size is quantified with Cohen's d.

\subsubsection{Electrical detection thresholds}


The mean $( \pm S D$ ) electrical detection threshold were $0.28 \pm 0.098$ in the high pain group and $0.26 \pm 0.08$ in the low pain group. No statistically significant difference was observed for the electrical detection thresholds between the two groups $(t(42)=-0.523, p=0.604$ Cohen's $d=-$ $0.158)$.

\subsubsection{Perceived mechanical pinprick intensity}

The mean and SD perceived mechanical pinprick intensity measured before and after HFS at each arm for both groups are shown in Figure 2.

The RM ANOVA revealed a significant TIME $\times \operatorname{SIDE}$ interaction $F_{G-G}(2.33,98.25)=45.31$, $p<0.001, \eta_{p}^{2}=0.519$ meaning that the mean perceived mechanical pinprick intensity was significantly different between the two arms. Post-hoc tests showed that the mean perceived pinprick intensity was not significantly different between the two arms at baseline (T0), but significantly differed after HFS at T1 ( $t=4.579, p<0.001 ; T 2 t=7.062, p<0.001 ; T 3 t=8.393$, $p<0.001$; and T4 $t=8.750, p<0.001$ ). These results confirm that HFS induced an increase in the perceived mechanical pinprick intensity.

The RM ANOVA also showed a significant TIME $\times$ SIDE $\times$ GROUP interaction $\left(F_{G-G}(2.33\right.$, $\left.98.25)=3.048, p=0.044, \eta_{p}{ }^{2}=0.068\right)$, which means that the difference in perceived mechanical pinprick intensity after HFS between the two arms was significantly different between the two groups. Post-hoc tests between the control and the HFS arm of the two groups (e.g. T1 $1_{\text {HFs }}$ and $\mathrm{T} 1_{\text {control }}$ of the high pain video vs. $\mathrm{T} 1_{\mathrm{HFS}}$ and $\mathrm{T} 1_{\text {control }}$ of the low pain video) were not statistically significant.

See Figure 2 and Table 2 for the details of the main results.

F test value pvalue Effect size

\begin{tabular}{|c|c|c|c|}
\hline & 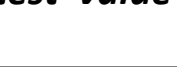 & & $\left(\eta^{2}{ }_{p}\right)$ \\
\hline Time & 8.569 & $<0.001$ & 0.169 \\
\hline Time $x$ Group & 0.523 & 0.625 & 0.012 \\
\hline Side & 66.677 & $<0.001$ & 0.614 \\
\hline Side $\times$ Group & 0.573 & 0.453 & 0.013 \\
\hline Time $x$ Side & 45.312 & $<0.001$ & 0.519 \\
\hline Time $x$ Side $x$ Group & 3.048 & 0.044 & 0.068 \\
\hline Group & 0.009 & 0.923 & $<0.001$ \\
\hline
\end{tabular}


Table 2. Results of the 3-way ANOVA on the perceived intensity of mechanical stimuli. Significant $p$-values are highlighted in red. Because the sphericity assumption was violated, the Greenhouse-Geisser correction was applied. Effect sizes are indicated as partial eta square.

\subsubsection{Perceived mechanical pinprick unpleasantness}

The mean and SD perceived mechanical pinprick unpleasantness measured before and after HFS at each arm for both groups are shown in Figure 2 (see Figure S1 for the individual data). The RM ANOVA revealed a significant TIME x SIDE interaction $\left(F_{G-G}(2.1,88.41)=36.7, p<0.001\right.$ $\left.\eta_{p}{ }^{2}=0.466\right)$, meaning that mechanical pinprick stimulation was perceived as more unpleasant on the HFS arm than on the control arm. Post-hoc tests showed that the participants did not report pinprick stimuli to be more unpleasant on the HFS arm compared to the control arm during baseline, but this difference emerged at T1 and remained significant during T2, T3, and T4 T1 $t=4.579 p<0.001 ; T 2=7.062 p<0.001 ; T 3 t=8.393 p<0.001 ;$ and T4 $t=8.750 p<0.001$. These results confirm that HFS induced an increase in the perceived mechanical pinprick unpleasantness.

The RM ANOVA did not show a significant TIME $\times$ SIDE $\times$ GROUP interaction $\left(F_{G-G}(2.1\right.$, 88.41)=1.511, $\left.p=0.226, \eta_{p}^{2}=0.035\right)$, meaning participants in the two groups did not differ in the perceived unpleasantness of mechanical stimulation on the two arms after HFS.

Table 3 reports the full statistics, Figure 3 a graphical representation.

\begin{tabular}{|c|c|c|c|}
\hline & F test value & $p$ value & $\begin{array}{c}\text { Effect size } \\
\left(\eta^{2}{ }_{p}\right)\end{array}$ \\
\hline Time & 15.513 & $<0.001$ & 0.270 \\
\hline Time x Group & 2.471 & 0.068 & 0.056 \\
\hline Side & 47.608 & $<0.001$ & 0.531 \\
\hline Side $\times$ Group & 0.453 & 0.504 & 0.002 \\
\hline Time $x$ Side & 36.698 & $<0.001$ & 0.466 \\
\hline Time $x$ Side $x$ Group & 1.511 & 0.226 & 0.035 \\
\hline Group & 0.646 & 0.426 & 0.015 \\
\hline
\end{tabular}

Table 3. Results of the 3-way ANOVA on the perceived unpleasantness of mechanical stimuli. Significant p-values are highlighted in red. Effect sizes are reported as partial eta square. 
Ratings of intensity

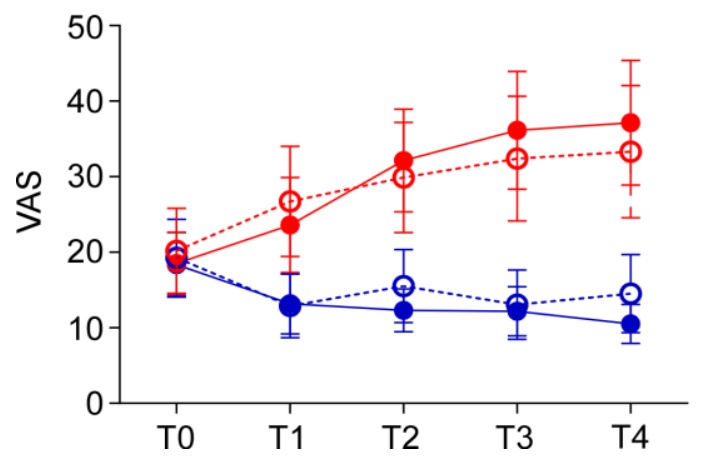

Ratings of unpleas

High Pain Video

- Control arm

- HFS arm

Low Pain Video

- Control arm

$\odot \cdot$ HFS arm

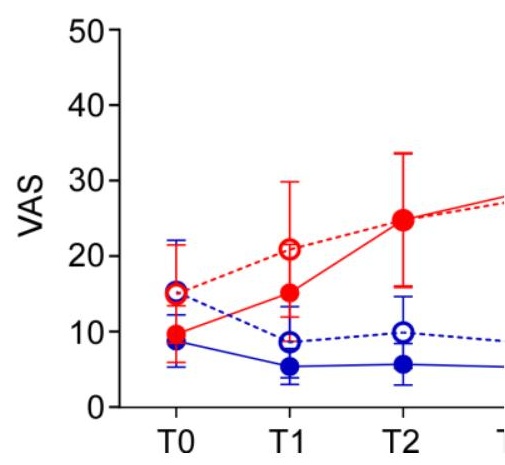

Figure 2. Mean and SD of the ratings of perceived intensity and unpleasantness for the two groups.

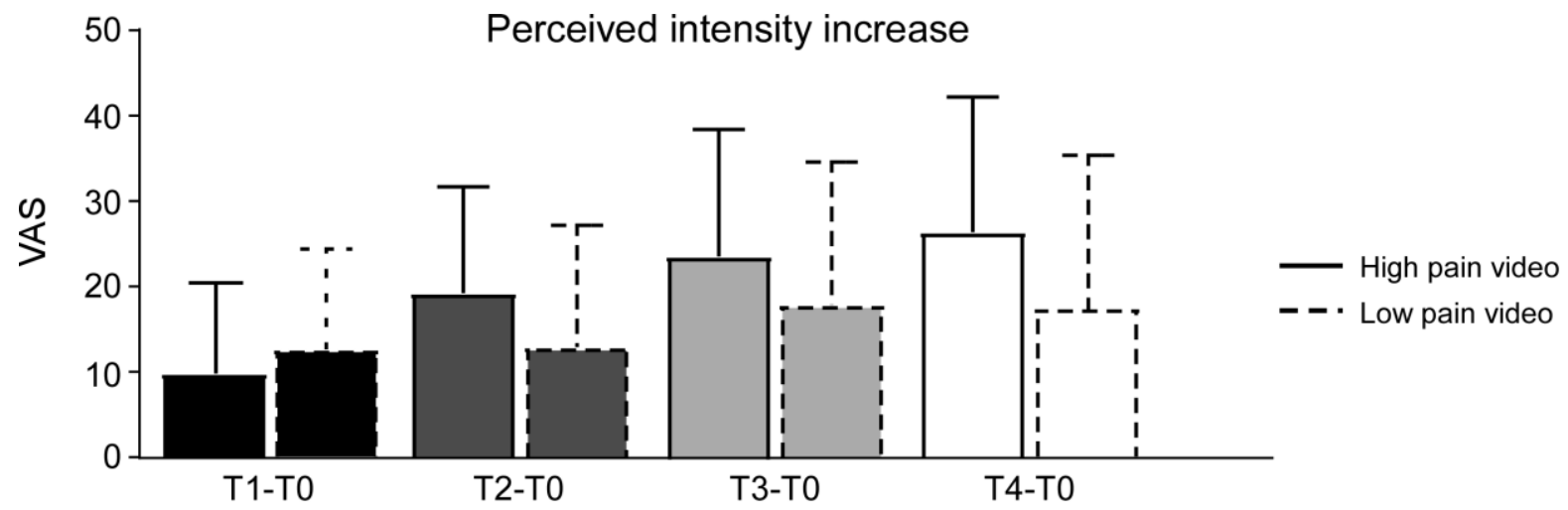

Perceived unpleasantness increase

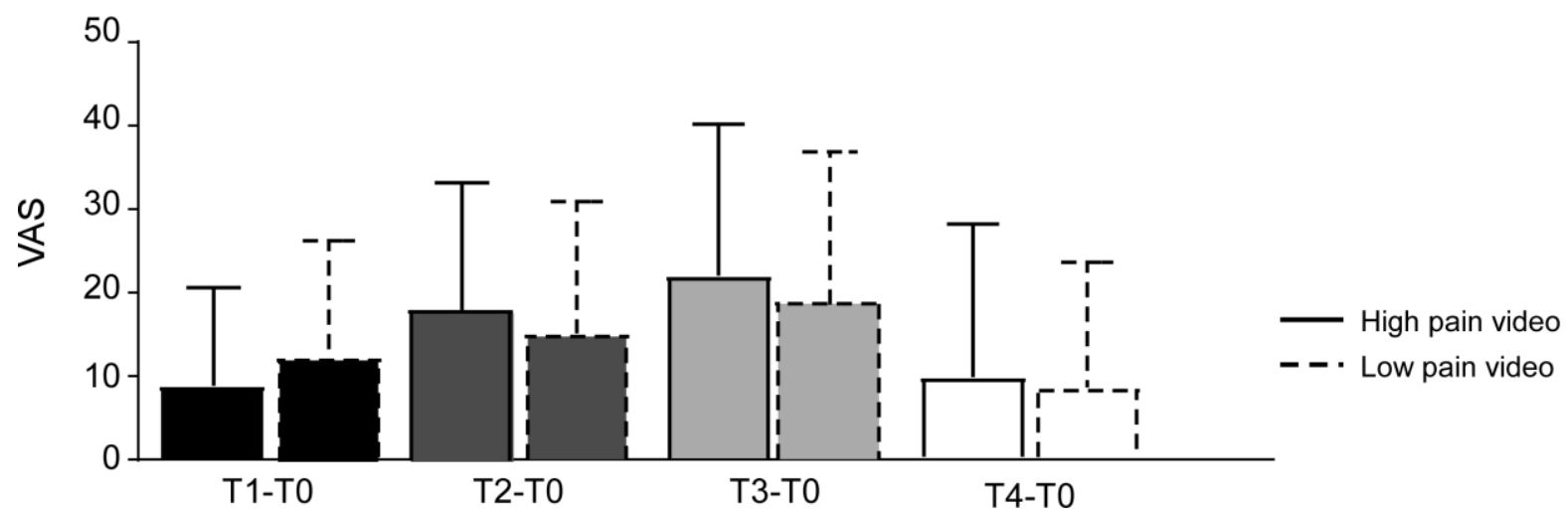


Figure 3. Increase in perceived intensity and unpleasantness of pinprick stimul applied at the stimulated arm at each time point in the two groups, after accounting for the ratings obtained for stimuli on the control arm (substraction HFS arm-Control arm per each time point).

\subsubsection{Spread of the area of increased pinprick sensitivity}

The mean and SD proximal-distal length of increased pinprick sensitivity were for the high pain video group $11.24 \pm 2.2 \mathrm{~cm}$ and for the low pain group $10.53 \pm 3 \mathrm{~cm}$. An independent t-test did not show a statistically significant difference in the proximal-distal length between the two groups $(t(42)=-0.879, p=0.192$, Cohen's $d=-0.265)$. See Figure 3.

\section{Vertical spread hyperalgesia}

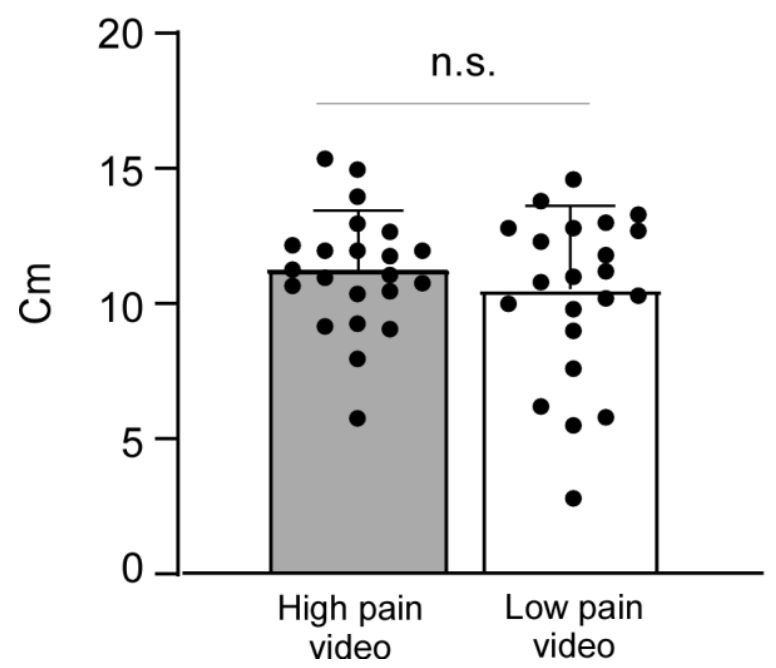

Figure 4. Mean and standard deviation of the length of the area of mechanical hypersensitivity in the two groups. Dots represent individual subjects.

\subsubsection{Perceived pain intensity during HFS}

The mean \pm SD pain ratings were $85.03 \pm 10.27$ for the high pain video group and $79.44 \pm 8.22$ for the low pain video group. The one sided t-test revealed that participants in the high pain 
group perceived HFS as more intense on average than the low pain group $t(42)=-1.992$ $p=0.026$, Cohen's $d=-0.601$.

Figure 5 shows the mean and SD and individual perceived pain intensity scores elicited by HFS.

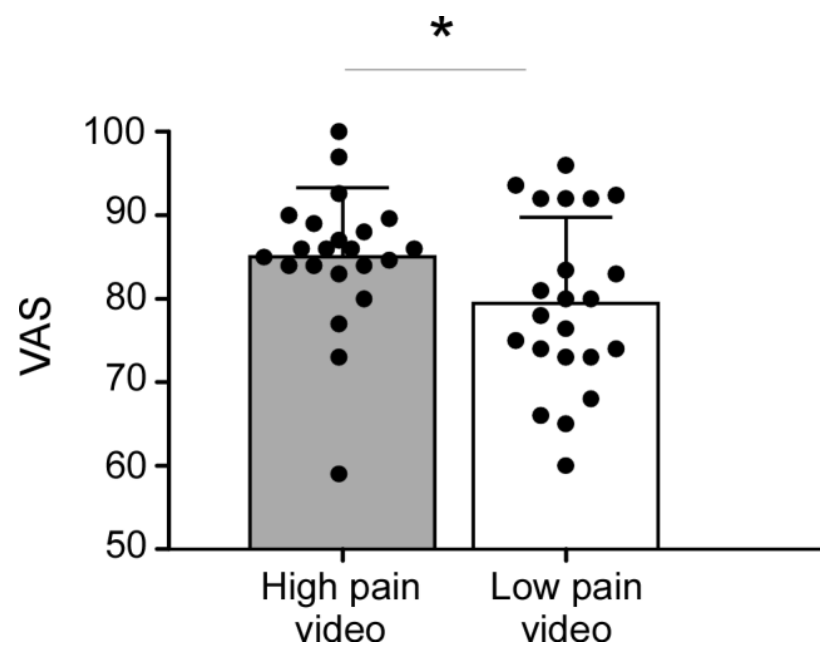

Figure 5. Mean and standard deviation of the perceived HFS intensity during the stimulation. The values in the two groups differed significantly * denotes a $p<0.05$.

\subsubsection{Exit questionnaire}

Contrary to our hypothesis, we did not observe a difference in the reported fear in the two groups $\mathrm{U}=189, \mathrm{p}=0.170$. In contrast, the high pain group reported a greater influence of painfulness scores reported by the actress on their pain scores $U=129.5 p=0.008$. There was no difference in the credibility scores between the two groups $U=283.5 p=0.320$.

\subsubsection{Correlation between fear of HFS and hypersensitivity}

There was no statistically significant correlation between fear of HFS and hypersensitivity, at any of the time points for intensity $\left(r_{s \mathrm{~T} 1-\mathrm{T} 0}=0.192 \mathrm{p}=0.211, \mathrm{r}_{\mathrm{s} \text { T2-T0}}=0.200 \mathrm{p}=0.193, \mathrm{r}_{\mathrm{s} \text { T3-T0 }}=0.201\right.$ $p=0.190, r_{s}$ T4-T0 $\left.=0.034 p=0.825\right)$. In contrast, whereas fear ratings did not correlate with hypersensitivity at $\mathrm{T} 1\left(r_{\mathrm{sT} 1-\mathrm{T} 0}=0.194 \mathrm{p}=0.208\right)$ and at T4 $\left(\mathrm{r}_{\mathrm{s}} \mathrm{T} 4-\mathrm{TO}=-0.091 \mathrm{p}=0.559\right)$, they did at $\mathrm{T} 2\left(r_{\mathrm{s}} \mathrm{T} 2-\mathrm{T} O_{0}=0.375 \mathrm{p}=0.012\right)$ and T3 $\left(\mathrm{r}_{\mathrm{s}} \mathrm{T3}-\mathrm{T} 0=0.399 \mathrm{p}=0.007\right)$. Both values survived a Bonferroni correction for 4 comparisons.

\subsubsection{Correlation between empathy and fear}


Contrary to experiment 1 , there was no statistically significant correlation between the total IRI scores and fear of the HFS stimulation $\left(r_{s}=0.121, p=0.433\right)$.

\subsubsection{Correlation perceived intensity of HFS and hypersensitivity}

The perceived intensity of HFS correlated positively with hypersensivity scores (intensity ratings) at T2, T3 and T4 ( $r_{\text {ST2-T0 }}=0.417 p=0.005, r_{s}$ T3-T0 $=0.483 p<0.001, r_{s}$ T4-T0 $\left.=0.487 p<0.001\right)$, but not at $\mathrm{T} 1\left(\mathrm{r}_{\mathrm{s}} \mathrm{T4}-\mathrm{TO}=0.134 \mathrm{p}=0.387\right)$. Unpleasantness scores correlated positively at $\mathrm{T} 2$ and T3 $\left(r_{s}\right.$ T2-T0 $=0.351 p=0.020, r_{s}$ T3-T0 $\left.=0.418 p=0.005\right)$, but not at T1 and T4 $\left(r_{\text {s T1-T0 }}=0.243 p=0.113\right.$, $\left.r_{\text {S T4-T0 }}=0.045 p=0.770\right)$.

\section{Discussion}

The present study was designed to investigate whether observing a model expressing high pain during HFS would lead to higher intensity pain ratings during HFS and more pronounced mechanical pinprick hypersensitivity, both in terms of intensity and unpleasantness, and in a larger spread. More specifically, we hypothesized that watching a video of a person expressing high pain during HFS would induce more fear of HFS as compared to watching a person showing less pain during HFS. We postulated that this increased fear would in turn lead to higher pain ratings for HFS and to a higher magnitude and a larger spread of pinprick hypersensitivity induced by HFS. Our hypotheses were partially confirmed.

\section{Observing a model expressing high pain increases one's own pain experience}

We found that the HFS trains were perceived on average as more intense in the high pain group compared to the low pain group. The significant difference in average pain rating to HFS between the two groups indicates that our manipulation was effective. However, and in contrast to the results of experiment 1 , we did not find a significant difference in fear of HFS between the two groups. This result raises doubts as to whether higher average pain ratings during HFS were mediated by differences in fear.

\section{Observing a model expressing high pain increases pinprick hypersensitivity induced by HFS}

We found that the increase in perceived intensity to mechanical pinprick stimuli applied after HFS was larger in the high pain group compared to low pain group. This finding suggests that observing a model expressing very intense pain during HFS, will not only increase one's own pain during HFS but will also facilitate the pinprick hypersensitivity after HFS (central 
sensitization). However, the effect size was small which, together with the fact that no followup test was found significant, indicates that in order to observe these effects future studies require much larger samples.

Surprisingly, no effects were observed on the perceived unpleasantness of the mechanical pinprick stimulation after HFS and the length of the area of pinprick hypersensitivity. It is possible that the lack of effects on the perceived unpleasantness ratings is underpinned by the large variability across participants.

Importantly, the present study used a novel design to investigate the effects of observational learning. Indeed, we opted for manipulating only the information regarding the painfulness of the HFS procedure, without instructing the participants about any potential change in the perceived intensity/unpleasantness of the pinprick stimulation. This was done in a previous study by van den Broeke et al., (24) in which the authors showed that negative expectations about the pinprick stimulation after HFS (i.e. that it would become painful) increased the development of HFS-induced pinprick hypersensitivity. Also notably, we opted to compare two pain conditions differing on the level of pain demonstrated by the model, rather than a more neutral control.

Observing a dissociation between intensity and unpleasantness is not common in pain studies (for a systematic review see (48)). Nevertheless, it has to be noted that such differences were mainly reported for studies targeting acute pain but not for studies assessing the hypersensitivity induced by acute pain. We might speculate that the lack of significant effects on unpleasantness scores was related to the lack of statistically significant difference in fear reported by the two groups. Of note, a lack of significant difference in fear scores between the two groups could also account for the statistically absent difference across the time points. Another possibility is that, given the large variability in unpleasantness scores, even larger samples would be required to observe a significant effect of group on unpleasantness scores (the triple interaction Time $\times$ Side $\times$ Group). Importantly however, the significant positive correlations that we observed between the perceived intensity of the HFS procedure and hypersensitivity would suggest that indeed an increase in the perceived intensity of HFS leads to greater hyperalgesia. Such an effect replicates what we had already observed in a previous study, in which we used a different model to induce hypersensitivity (e.g. Low Frequency Stimulation of the skin, LFS) (46). More than the actual intensity of stimulation, the perceived 
intensity seems to be related to the increase in hypersensitivity, confirming the role of topdown modulatory factors

The lack of differences in the length of the area of pinprick hypersensitivity could be related to the use of a too high stimulation intensity for HFS ( $20 \times$ detection threshold) that resulted in a ceiling effect in the spread of pinprick hypersensitivity.

\section{The role of fear and empathy}

While in experiment 1 the high pain video was associated with higher fear ratings, the statistical comparison in fear levels in experiment 2 failed to reach statistical significance. This results did not depend on the sample size as the same results were obtained in experiment 1 when just a subset of participants was selected in order to match the required numerosity of experiment 2. However, one substantial difference is that in experiment 2 the fear scores were obtained at the end of the experiment, after participants had experienced HFS, whereas in experiment 1 they were obtained after watching the videos as no HFS was actually administered. It is therefore possible that in experiment 2 some participants made adjustments to their scores based on the actual experience. This would be also consistent with the results of Vögtle et al., (16), who did not find any correlation between expectations and nocebo effects when the ratings were asked retrospectively.

Whether fear is potentially the main drive of differential effects based on observation remains therefore an open question. In experiment 2, fear ratings did not correlate with hypersensitivity, nor did the empathy scores. Of note, we used a Likert scale from 0 to 5 for fear ratings, and it can be argued that as such we potentially lost the necessary variability to disclose more subtle relationships. On the other hand, we did find a significant relationship between fear and pinprick ratings. In this sense, it may be argued that, in this sample and with this experimental manipulation, the effects of fear were generalized to all pinprick stimuli, and not specifically associated with hyperalgesia.

The role that empathy has in observational learning is more controversial. Previous studies have found significant (9), but mainly non-significant effects of empathy scores on placebo/nocebo effects imparted by observational learning $(7,16,17)$. It has been proposed (16) that the specific experimental conditions, namely the in person presence of the model or the presentation of a recorded video, may be part of the explanation. However, it should also 
be noticed that also Swider and Babel (9) discuss the relationship between empathy and nocebo effects cautiously as their results did not provide the strong support that had been initially proposed (3).

\section{Conclusion}

Our present findings suggest that observing a person experiencing very intense pain during HFS may increase not only one's own pain experience during HFS but also the consequences of the pain, i.e. development of central sensitization manifested as pinprick hypersensitivity. However, these effects appear to be small and in order to be detected future studies need much larger sample sizes and/or stronger fear inductions. It remains to be elucidated through which mechanisms observational learning modulates the development of pinprick hypersensitivity. 


\section{Bibliography}

1. Bandura A. Social foundations of thought and action. The health psychology reader. 1 Oliver's Yard, 55 City Road, London EC1Y 1SP United Kingdom : SAGE Publications Ltd; 2002. p. 94-106.

2. Goubert L, Vlaeyen JWS, Crombez G, Craig KD. Learning about pain from others: an observational learning account. J Pain. 2011 Feb;12(2):167-174.

3. Colloca L, Benedetti F. Placebo analgesia induced by social observational learning. Pain. 2009 Jul;144(1-2):28-34.

4. Helsen K, Goubert L, Peters ML, Vlaeyen JWS. Observational learning and pain-related fear: an experimental study with colored cold pressor tasks. J Pain. 2011 Dec;12(12):1230-1239.

5. Helsen K, Vlaeyen JWS, Goubert L. Indirect acquisition of pain-related fear: an experimental study of observational learning using coloured cold metal bars. PLoS One. 2015 Mar 25;10(3):e0117236.

6. Bajcar EA, Bąbel P. How does observational learning produce placebo effects? A model integrating research findings. Front Psychol. 2018 Oct 24;9:2041.

7. Vögtle E, Barke A, Kröner-Herwig B. Nocebo hyperalgesia induced by social observational learning. Pain. 2013 Aug;154(8):1427-1433.

8. Helsen K, Goubert L, Vlaeyen JWS. Observational learning and pain-related fear: exploring contingency learning in an experimental study using colored warm water immersions. J Pain. 2013 Jul;14(7):676-688.

9. Swider K, Bąbel P. The effect of the sex of a model on nocebo hyperalgesia induced by social observational learning. Pain. 2013 Aug;154(8):1312-1317.

10. Świder K, Bąbel P. The effect of the type and colour of placebo stimuli on placebo effects induced by observational learning. PLoS One. 2016 Jun 30;11(6):e0158363.

11. Trost Z, France CR, Vervoort T, Lange JM, Goubert L. Learning about pain through observation: the role of pain-related fear. J Behav Med. 2014 Apr;37(2):257-265.

12. Olsson A, Knapska E, Lindström B. The neural and computational systems of social learning. Nat Rev Neurosci. 2020 Mar 12;21(4):197-212.

13. Bootzin RR, Caspi O. Explanatory mechanisms for placebo effects: cognition, personality and social learning. The science of the placebo: Toward an .... 2002;

14. Schenk LA, Krimmel SR, Colloca L. Observe to get pain relief: current evidence and potential mechanisms of socially learned pain modulation. Pain. 2017 Nov;158(11):2077-2081. 
15. Egorova N, Park J, Orr SP, Kirsch I, Gollub RL, Kong J. Not seeing or feeling is still believing: conscious and non-conscious pain modulation after direct and observational learning. Sci Rep. 2015 Nov 18;5:16809.

16. Vögtle $E$, Kröner-Herwig B, Barke A. Nocebo hyperalgesia can be induced by the observation of a model showing natural pain expressions. Clin J Pain. 2019;35(9):737743.

17. Vögtle E, Kröner-Herwig B, Barke A. Nocebo hyperalgesia: contributions of social observation and body-related cognitive styles. J Pain Res. 2016 Apr 22;9:241-249.

18. Keum S, Shin H-S. Neural basis of observational fear learning: A potential model of affective empathy. Neuron. 2019 Oct 9;104(1):78-86.

19. Loeser JD, Treede R-D. The Kyoto protocol of IASP Basic Pain Terminology. Pain. 2008 Jul 31;137(3):473-477.

20. Woolf CJ. Central sensitization: implications for the diagnosis and treatment of pain. Pain. 2011 Mar;152(3 Suppl):S2-15.

21. Latremoliere A, Woolf CJ. Central sensitization: a generator of pain hypersensitivity by central neural plasticity. J Pain. 2009 Sep;10(9):895-926.

22. Olsson A, Nearing KI, Phelps EA. Learning fears by observing others: The neural systems of social fear transmission. Soc Cogn Affect Neurosci. 2007 Mar;2(1):3-11.

23. Campbell JID, Thompson VA. MorePower 6.0 for ANOVA with relational confidence intervals and Bayesian analysis. Behav Res Methods. 2012 Dec;44(4):1255-1265.

24. van den Broeke EN, Geene N, van Rijn CM, Wilder-Smith OHG, Oosterman J. Negative expectations facilitate mechanical hyperalgesia after high-frequency electrical stimulation of human skin. Eur J Pain. 2014 Jan;18(1):86-91.

25. van den Broeke EN, van Heck CH, Ceelen LAJM, van Rijn CM, van Goor H, Wilder-Smith OHG. The effect of high-frequency conditioning stimulation of human skin on reported pain intensity and event-related potentials. J Neurophysiol. 2012 Oct;108(8):22762281.

26. van den Broeke EN, Mouraux A. High-frequency electrical stimulation of the human skin induces heterotopical mechanical hyperalgesia, heat hyperalgesia, and enhanced responses to nonnociceptive vibrotactile input. J Neurophysiol. 2014 Apr;111(8):15641573.

27. van den Broeke EN, Lambert J, Huang G, Mouraux A. Central Sensitization of Mechanical Nociceptive Pathways Is Associated with a Long-Lasting Increase of Pinprick-Evoked Brain Potentials. Front Hum Neurosci. 2016 Oct 20;10:531.

28. van den Broeke EN, de Hemptinne P, Mercken M, Torta DM, Lambert J, Mouraux A. Central sensitization of nociceptive pathways demonstrated by robot-controlled pinprick-evoked brain potentials. Clin Neurophysiol. 2020 Oct;131(10):2491-2498. 
29. van den Broeke EN, Hartgerink DM, Butler J, Lambert J, Mouraux A. Central sensitization increases the pupil dilation elicited by mechanical pinprick stimulation. J Neurophysiol. 2019 May 1;121(5):1621-1632.

30. van den Broeke EN, de Vries B, Lambert J, Torta DM, Mouraux A. Phase-locked and non-phase-locked EEG responses to pinprick stimulation before and after experimentally-induced secondary hyperalgesia. Clin Neurophysiol. 2017 May 19;128(8):1445-1456.

31. van den Broeke EN, Mouraux A, Groneberg AH, Pfau DB, Treede R-D, Klein T. Characterizing pinprick-evoked brain potentials before and after experimentally induced secondary hyperalgesia. J Neurophysiol. 2015 Nov;114(5):2672-2681.

32. van den Broeke EN, Gousset S, Bouvy J, Stouffs A, Lebrun L, van Neerven SGA, et al. Heterosynaptic facilitation of mechanical nociceptive input is dependent on the frequency of conditioning stimulation. J Neurophysiol. 2019 Sep 1;122(3):994-1001.

33. Vo L, Drummond PD. Analgesia to pressure-pain develops in the ipsilateral forehead after high- and low-frequency electrical stimulation of the forearm. Exp Brain Res. 2014 Feb;232(2):685-693.

34. Vo L, Hood S, Drummond PD. Involvement of Opioid Receptors and $\alpha 2$-Adrenoceptors in Inhibitory Pain Modulation Processes: A Double-Blind Placebo-Controlled Crossover Study. J Pain. 2016 Nov;17(11):1164-1173.

35. Vo L, Drummond PD. Big girls don"t cry': the effect of the experimenter"s sex and pain catastrophising on pain. Scand J Pain. 2021 Jul 27;21(3):617-627.

36. Davis $\mathrm{MH}$. Measuring individual differences in empathy: Evidence for a multidimensional approach. J Pers Soc Psychol. 1983;44(1):113-126.

37. De Corte K, Buysse A, Verhofstadt LL, Roeyers H, Ponnet K, Davis MH. Measuring empathic tendencies: reliability and validity of the dutch version of the interpersonal reactivity index. Psychol Belg. 2007 Oct 1;47(4):235.

38. Spielberger CD, Jacobs G, Russell S. Assessment of anger: The state-trait anger scale. Advances in personality .... 1983;

39. Van der Ploeg HM. Validity of the Zelf-Beoordelings-Vragenlijst (A Dutch version of the Spielberger State-Trait Anxiety Inventory). Nederlands Tijdschrift voor de Psychologie en .... 1980;

40. Fredrickson BL, Tugade MM, Waugh CE, Larkin GR. What good are positive emotions in crisis? A prospective study of resilience and emotions following the terrorist attacks on the United States on September 11th, 2001. J Pers Soc Psychol. 2003;84(2):365-376.

41. Sullivan MJL, Bishop SR, Pivik J. The Pain Catastrophizing Scale: Development and validation. Psychol Assess. 1995;7(4):524-532. 
42. Van Damme S, Crombez G, Vlaeyen J. De pain catastrophizing scale: psychometrische karakteristieken en normering. 2000;

43. McNeil DW, Rainwater AJ. Development of the Fear of Pain Questionnaire--III. J Behav Med. 1998 Aug;21(4):389-410.

44. Roelofs J, Peters ML, Deutz J, Spijker C, Vlaeyen JWS. The Fear of Pain Questionnaire (FPQ): further psychometric examination in a non-clinical sample. Pain. 2005 Aug;116(3):339-346.

45. van den Broeke EN, van Rijn CM, Biurrun Manresa JA, Andersen OK, Arendt-Nielsen L, Wilder-Smith OHG. Neurophysiological correlates of nociceptive heterosynaptic longterm potentiation in humans. J Neurophysiol. 2010 Apr;103(4):2107-2113.

46. Torta DM, De Laurentis M, Eichin KN, von Leupoldt A, van den Broeke EN, Vlaeyen JWS. A highly cognitive demanding working memory task may prevent the development of nociceptive hypersensitivity. Pain. 2020;161(7):1459-1469.

47. Cayrol T, Lebleu J, Mouraux A, Roussel N, Pitance L, van den Broeke EN. Within- and between-session reliability of secondary hyperalgesia induced by electrical highfrequency stimulation. Eur J Pain. 2020 Jun 30;24(8):1585-1597.

48. Talbot K, Madden VJ, Jones SL, Moseley GL. The sensory and affective components of pain: are they differentially modifiable dimensions or inseparable aspects of a unitary experience? A systematic review. Br J Anaesth. 2019 Aug;123(2):e263-e272.

53. van den Broeke EN, Geene N, van Rijn CM, Wilder-Smith OHG, Oosterman J. Negative expectations facilitate mechanical hyperalgesia after high-frequency electrical stimulation of human skin. Eur J Pain. 2014 Jan;18(1):86-91.

54. Matre D, Casey KL, Knardahl S. Placebo-induced changes in spinal cord pain processing. J Neurosci. 2006 Jan 11;26(2):559-563.

55. Geuter S, Büchel C. Facilitation of pain in the human spinal cord by nocebo treatment. J Neurosci. 2013 Aug 21;33(34):13784-13790.

56. Tinnermann A, Geuter S, Sprenger C, Finsterbusch J, Büchel C. Interactions between brain and spinal cord mediate value effects in nocebo hyperalgesia. Science. 2017 Oct 6;358(6359):105-108.

57. Talbot K, Madden VJ, Jones SL, Moseley GL. The sensory and affective components of pain: are they differentially modifiable dimensions or inseparable aspects of a unitary experience? A systematic review. Br J Anaesth. 2019 Aug;123(2):e263-e272. 